\title{
Exotic Quarkonium Spectroscopy and Production
}

\author{
E. Asli Yetkin ${ }^{* \dagger}$ \\ University of Iowa, USA \\ Mimar Sinan Fine Arts University, TURKEY \\ E-mail: eaa@cern.ch
}

Using large data samples of di-muon events collected at $\sqrt{s}=7 \mathrm{TeV}$ center of mass energy, CMS has performed detailed measurements in the field of exotic quarkonium production. The production of $\mathrm{X}(3872)$ via decays to $J / \psi \pi^{+} \pi^{-}$is studied measuring the ratio of the $\mathrm{X}(3872)$ and $\psi(2 S)$ cross section times their branching fraction as a function of $J / \psi \pi^{+} \pi^{-}$transverse momentum $\left(p_{T}\right)$. Additionally the fraction of $\mathrm{X}(3872)$ from the decays of the $B$ hadrons is determined first time at Large Hadron Collider (LHC) and prompt X(3872) differential cross section times branching fraction as a function of $p_{T}$ is measured for the first time ever. The dipion invariant mass spectrum from the $J / \psi \pi^{+} \pi^{-}$system in the X(3872) decay is also investigated to establish the nature and the details of this decay.

XXI International Workshop on Deep-Inelastic Scattering and Related Subject -DIS2013, 22-26 April 2013

Marseilles, France

\footnotetext{
*Speaker.

${ }^{\dagger}$ On behalf of the CMS Collaboration
} 


\section{Introduction}

The observation of new states which do not fit into the conventional quark model renewed the interest in the exotic states. Many models such as hybrid mesons, tetra-quark states and the molecular states are proposed to explain the charmonium spectroscopy, however the agreement with experimental picture is not satisfied. To be able to understand the nature of these new states more experimental input is needed. Using $\sqrt{s}=7 \mathrm{TeV}$ pp collision data, the initial exotic spectroscopy program followed by CMS included the measurement of $\mathrm{X}(3872)$ production via decays to $J / \psi \pi^{+} \pi^{-}$

\section{Production of $\mathrm{X}(3872)$}

Since the discovery of X(3872) by Belle [1] in 2003 many studies have been performed to understand the production mechanism of $\mathrm{X}(3872)$, however, its nature is still unclear. Using the $4.8 \mathrm{fb}^{-1}$ pp collisions data collected in 2011 at $\sqrt{s}=7 \mathrm{TeV}$, CMS studied the production of $\mathrm{X}$ (3872) by measuring the cross sections times branching fraction, the non-prompt fraction, prompt cross section, and also the invariant mass spectrum of $\pi^{+} \pi^{-}$from X(3872) to $J / \psi \pi^{+} \pi^{-}$decay. The subsections 2.1, 2.2, 2.3, and 2.4 will summarize the results of these measurement where information on the event selection and details of the measurements can be found in Ref. [2].

\subsection{Measurement of the cross section ratio}

The ratio of the cross section times the $J / \psi \pi^{+} \pi^{-}$branching fraction is measured from the $\mathrm{X}(3872)$ and $\psi(2 S)$ signal events correcting for the acceptance and the efficiency which are estimated from simulation.

$$
R=\frac{\sigma(p p \rightarrow X(3872)+\text { anyt. }) \cdot \mathscr{B}\left(X(3872) \rightarrow J / \psi \pi^{+} \pi^{-}\right)}{\sigma(p p \rightarrow \psi(2 S)+\text { anyt. }) \cdot \mathscr{B}\left(\psi(2 S) \rightarrow J / \psi \pi^{+} \pi^{-}\right)}=\frac{N_{X(3872)} \cdot A_{\psi(2 S)} \cdot \varepsilon_{\psi(2 S)}}{N_{\psi(2 S)} \cdot A_{X(3872)} \cdot \varepsilon_{X(3872)}}
$$

The signal events for $\mathrm{X}(3872)$ and $\psi(2 S)$ are separately extracted from the unbinned maximumlikelihood fits to the $J / \psi \pi^{+} \pi^{-}$invariant mass spectrum. In the fits the signal for the $\psi(2 S)$ resonance is modeled as two Gaussian functions with a common mean, and as a single Gaussian function for the $\mathrm{X}(3872)$. The non-resonant background is modeled as a second order Chebyshev polynomial. Acceptance and efficiencies are estimated from simulation, assuming both $\mathrm{X}(3872)$ and $\psi(2 S)$ are unpolarized and $J^{P C}$ of $\mathrm{X}(3872)=1^{++}$as recently measured by $\mathrm{LHCb}[3]$. The cross section times the branching fraction measurement is also presented as "fiducial" measurement (w/o acceptance correction) within a phase space window defined by the kinematical cuts on the muon and pion pairs $\left(p_{T}^{\mu}>4 \mathrm{GeV}\right.$ in $\left|\eta^{\mu}\right|<1.2, p_{T}^{\mu}>3.3 \mathrm{GeV}$ in $1.2<\left|\eta^{\mu}\right|<2.4, p_{T}^{\mu \mu}>7 \mathrm{GeV}$ in $\left.\left|y^{\mu \mu}\right|<1.25, p_{T}^{\pi}>0.6 \mathrm{GeV} \& \Delta R(\pi, \mu \mu)<0.55\right)$. The cross section times branching fraction ratio as a function of transverse momentum of the $J / \psi \pi^{+} \pi^{-}$systems are shown in Fig. 1 and listed in Table 1 for $R_{\text {fiducial }}\left(1^{\text {st }}\right.$ column) and the fully acceptance-corrected R ( $2^{\text {nd }}$ column). No significance dependence on the transverse momentum is observed. Measuring the ratio cancels some of the systematics which are common to both $\mathrm{X}(3872)$ and $\psi(2 S)$ measurements. The main systematic uncertainty in the cross section ratio arises from the limited knowledge of the $\mathrm{X}(3872)$ transverse momentum. 

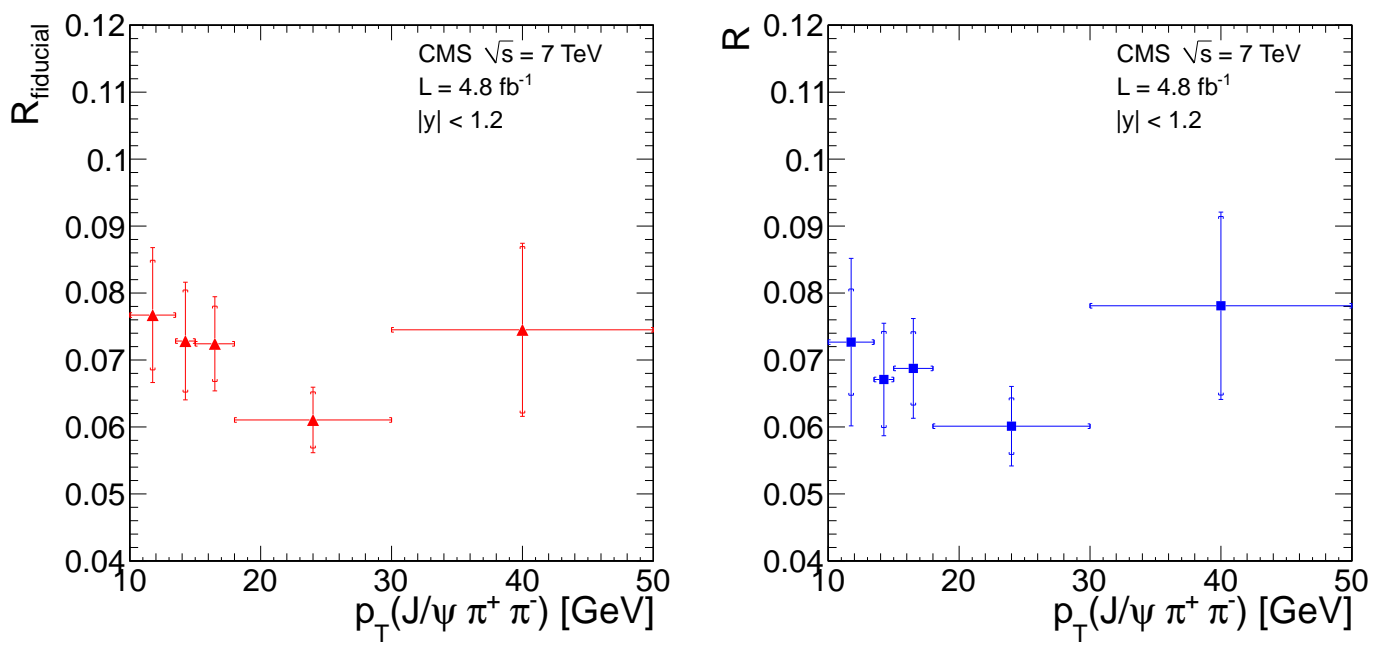

Figure 1: Ratios of the $\mathrm{X}(3872)$ and $\psi(2 \mathrm{~S})$ cross sections times branching fractions, without ( $\mathrm{R}_{\text {fiducial }}$, left) and with (R, right) acceptance corrections for the muon and pion pairs, as a function of $p_{T}$. The data points are placed at the centre of each $p_{T}$ bin. The inner error bars indicate the statistical uncertainty and the outer error bars represent the total uncertainty.

Table 1: The cross sections times branching fractions ratios $R_{\text {fiducial }}, \mathrm{R}$, and the non-prompt fraction of the $\mathrm{X}(3872)$ production as a function of $p_{T}$ for the rapidity range $|y|<1.2$. The shown uncertainties are statistical and systematic, respectively.

\begin{tabular}{|c|c|c|c|}
\hline$p_{T}(\mathrm{GeV})$ & $\mathrm{R}_{\text {fiducial }}$ & $\mathrm{R}$ & non-prompt fraction \\
\hline $10-13.5$ & $0.0767 \pm 0.0082 \pm 0.0059$ & $0.0727 \pm 0.0079 \pm 0.0097$ & $0.272 \pm 0.057 \pm 0.016$ \\
$13.5-15$ & $0.0728 \pm 0.0076 \pm 0.0044$ & $0.0671 \pm 0.0072 \pm 0.0044$ & $0.182 \pm 0.052 \pm 0.013$ \\
$15-18$ & $0.0724 \pm 0.0056 \pm 0.0042$ & $0.0687 \pm 0.0055 \pm 0.0051$ & $0.246 \pm 0.043 \pm 0.015$ \\
$18-30$ & $0.0611 \pm 0.0042 \pm 0.0025$ & $0.0601 \pm 0.0042 \pm 0.0042$ & $0.297 \pm 0.042 \pm 0.021$ \\
$30-50$ & $0.075 \pm 0.012 \pm 0.004$ & $0.078 \pm 0.013 \pm 0.004$ & $0.301 \pm 0.097 \pm 0.030$ \\
\hline $10-50$ & $0.0694 \pm 0.0029 \pm 0.0036$ & $0.0656 \pm 0.0029 \pm 0.0065$ & $0.263 \pm 0.023 \pm 0.016$ \\
\hline
\end{tabular}

\subsection{Measurement of the non-prompt production}

The fraction of the non-prompt $\mathrm{X}(3872)$ production is determined from the decay lifetime distribution. The "pseudo-proper" decay length $l_{x y}$ is defined in a plane transverse to the beam direction as the separation between $J / \psi \pi^{+} \pi^{-}$vertex and the closest reconstructed primary vertex along the beam direction and corrected by the Lorentz boost of the $J / \psi \pi^{+} \pi^{-}$candidate. The $B$ enriched sample of $\mathrm{X}(3872)$ candidates are selected by requiring $l_{x y}$ to be greater than $100 \mu \mathrm{m}$. The non-prompt fraction is calculated as the ratio of the $B$ hadron enriched sample signal yield and the signal yield in the inclusive sample and measured as a function of transverse momentum. The results are shown in the last column of Table 1 as a function of $p_{T}$ for the rapidity range $|y|<1.2$. No significance $p_{T}$ dependence is observed. The measurement is statistically dominated.

\subsection{Measurement of the prompt cross section}

The cross section times branching fraction for prompt $\mathrm{X}(3872)$ production is determined using 

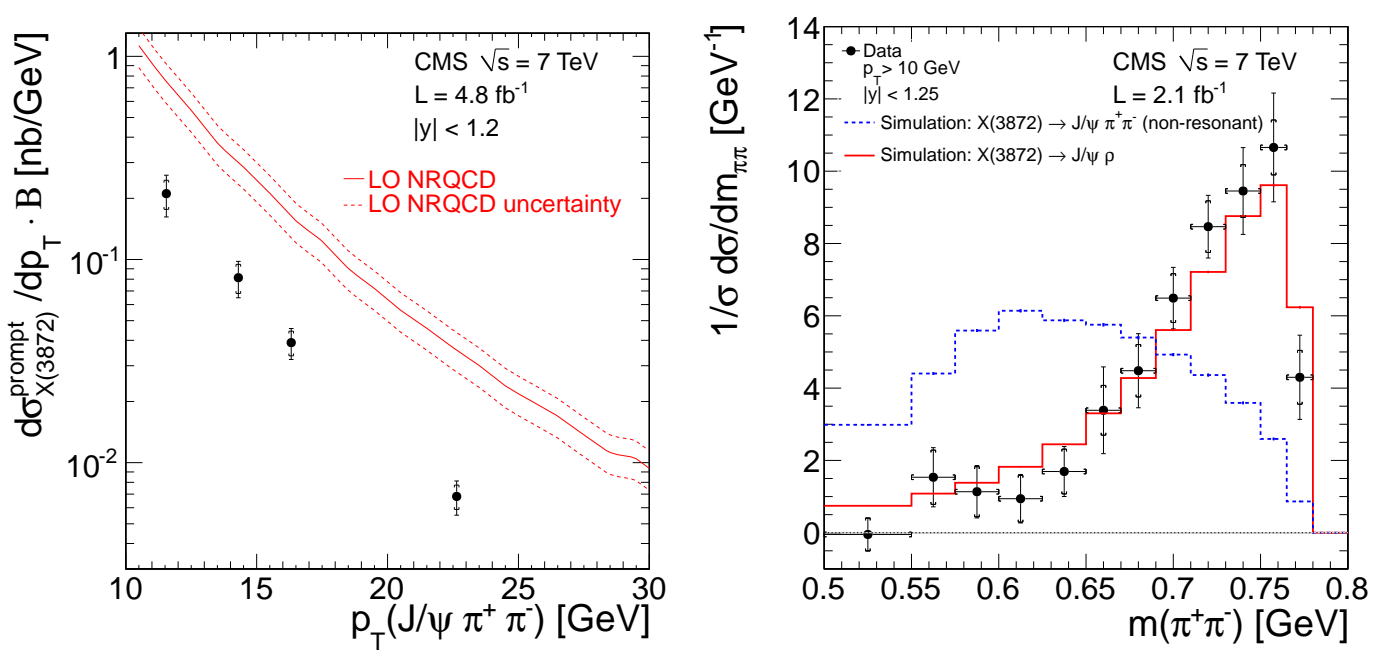

Figure 2: Left: Prompt $X(3872)$ production times branching fraction $\mathscr{B}\left(X(3872) \rightarrow J / \psi \pi^{+} \pi^{-}\right)$as a function of $p_{T}$. The inner error bars indicate the statistical uncertainty and the outer error bars indicate the total uncertainty. Right: the acceptance and efficiency corrected dipion invariant mass spectrum for $\mathrm{X}(3872)$ $\rightarrow J / \psi \pi^{+} \pi^{-}$. The distribution is normalized dividing by the total cross section for $0.5<\mathrm{m}\left(\pi^{+} \pi^{-}\right)<0.78$ $\mathrm{GeV}$. The results are compared to simulation with and without an intermediate $\rho^{0}$ decay.

the cross section ratio R (sec. 2.1), the non-prompt fraction (sec. 2.2), and the previous CMS measurement on the prompt $\psi(2 S)$ production [4] as a function of transverse momentum up to 30 $\mathrm{GeV}$ and for the rapidity range $|y|<1.2$. The measurement is compared with predicted differential cross section which was made using the NRQCD factorization formalism [5]. The predictions given in Ref. [5] are modified to be able to match the phase space conditions used in CMS X(3872) prompt cross section measurement. The comparison (Fig. 2 left) showed that although predictions describe the shape reasonably well, the predicted cross section is much larger than the observation made in data. CMS provided the integrated prompt $\mathrm{X}(3872)$ cross section times branching fraction for the kinematical region $10<p_{T}<30 \mathrm{GeV}$ and |y|< 1.2 as $1.06 \pm 0.11$ (stat.) \pm 0.15 (syst.) nb. The NRQCD prediction for prompt $\mathrm{X}(3872)$ production is $4.01 \pm 0.88 \mathrm{nb}$ [5] which is well above the CMS measurement. This important CMS measurement on the prompt cross section is recently quoted by many theoretical papers[6, 7, 8, 9].

\subsection{Measurement of the $\pi^{+} \pi^{-}$invariant mass distribution}

The invariant mass distribution of $\pi^{+} \pi^{-}$pair from $\mathrm{X}(3872) \rightarrow J / \psi \pi^{+} \pi^{-}$for the kinematical range $10<p_{T}<50 \mathrm{GeV}$ and $|\mathrm{y}|<1.25$ (Fig. 2 right) is measured to further investigate the decay properties of the $\mathrm{X}(3872)$. The data are compared to signal simulations with and without intermediate $\rho^{0}$ in the $J / \psi \pi^{+} \pi^{-}$decay. The study showed that the intermediate $\rho^{0}$ decay (X(3872) $\rightarrow J / \psi \rho^{0} \rightarrow \mu^{+} \mu^{-} \pi^{+} \pi^{-}$) gives better agreement with the data.

\section{Summary}

The exotic quarkonium sector studies performed by CMS experiment using large data samples of dimuon events collected at $\sqrt{s}=7 \mathrm{TeV}$ center of mass energy are summarized in this proceeding. 
The production of $\mathrm{X}(3872)$ via decays to $J / \psi \pi^{+} \pi^{-}$is studied by measuring the ratio of the $\mathrm{X}(3872)$ and $\psi(2 S)$ cross section times branching fractions as function of transverse momentum in the rapidity range $|y|<1.2$. The non-prompt fraction of $\mathrm{X}(3872)$ (from the decays of $B$ hadrons) is measured as $0.263 \pm 0.023$ (stat.) \pm 0.016 (syst.) and no significance $p_{T}$ dependence is observed. Assuming both $\mathrm{X}(3872)$ and $\psi(2 S)$ are unpolarized, CMS measured the prompt $\mathrm{X}(3872)$ production times branching fraction into $J / \psi \pi^{+} \pi^{-}$for the first time as a function of $p_{T}$. The NRQCD predictions overestimate the prompt X(3872) production at LHC where CMS measured $\sigma^{\text {prompt }}(p p \rightarrow X(3872)+$ anything $) \times \mathscr{B}\left(X(3872) \rightarrow J / \psi \pi^{+} \pi^{-}\right)$in the kinematical region $10<p_{T}<30 \mathrm{GeV}$ and $|y|<1.2$ as $1.06 \pm 0.11$ (stat.) \pm 0.15 (syst.) nb. The studies of dipion mass spectrum for $\mathrm{X}(3872)$ showed that $X(3872) \rightarrow J / \psi \pi^{+} \pi^{-}$favors the presence of an intermediate $\rho^{0}$ state.

\section{Acknowledgment}

Work of author E.Asli Yetkin in Mimar Sinan Fine Arts University is supported by TUBITAK (Bideb-2232) research grant.

\section{References}

[1] Belle Collaboration, Observation of a new narrow charmonium state in exclusive $B^{ \pm} \rightarrow K^{ \pm} \pi^{+} \pi^{-} J / \psi$ decays, Phys. Rev. Lett. 91 (262001) 2003.

[2] CMS Collaboration, Measurement of the X(3872) production cross section via decays to $J / \psi \pi^{+} \pi^{-}$in pp collisions at $\sqrt{s}=7$ TeV, JHEP 04 (154) 2013 [hep-ex/1302 . 3968].

[3] LHCb Collaboration, Determination of the X(3872) Meson Quantum Numbers, Phys. Rev. Lett. 110 222001 (2013)

[4] CMS Collaboration, $J / \psi$ and $\psi(2 S)$ production in pp collisions at $\sqrt{s}=7 \mathrm{TeV}$, JHEP 05 (026) 2012

[5] P. Artoisenet and E. Braaten, Production of the X(3872) at the Tevatron and the LHC, Phys. Rev. D 81 (114018) 2010

[6] C. Hidalgo-Duque, J. Nieves, A. Ozpineci, V. Zamiralov, X(3872) and its Partners in the Heavy Quark Limit of QCD, [tt hep-ex/1305.4487].

[7] A. Esposito, F. Piccinini, A. Pilloni, AD Polosa, A Mechanism for Hadron Molecule Production in $p \bar{p}(p)$ Collisions, [tt hep-ex/1305.0527].

[8] C. Meng, H. Han, K. Chao, X(3872) and its production at hadron colliders, [tt hep-ex/1304.6710]

[9] M. Butenschoen, Z. He, B.A. Kniehl, NLO NRQCD disfavors the interpretation of X(3872) as $\chi_{c 1}(2 P)$, [tt hep-ex/1303.6524] 